\title{
Osmoregulation in Polycystic Kidney Disease: Relationship with Cystogenesis and Hypertension
}

\author{
Conor F. Underwood Jacqueline K. Phillips Cara M. Hildreth \\ Department of Biomedical Sciences, Faculty of Medicine and Health Sciences, Macquarie University, Sydney, NSW, Australia
}

\section{Keywords}

Polycystic kidney disease - Vasopressin . Sympathetic nervous system $\cdot$ Blood pressure $\cdot$ Hypertension .

Osmolality · Urinary concentrating ability

\begin{abstract}
Polycystic kidney disease (PKD) is a group of monogenetic conditions characterised by the progressive accumulation of multiple renal cysts and hypertension. One of the earliest features of PKD is a reduction in urinary concentrating capacity that impairs extracellular fluid conservation. Urinary concentrating impairment predisposes PKD patients to periods of hypohydration when fluid loss is not adequately compensated by fluid intake. The hypohydrated state provides a blood hyperosmotic stimulus for vasopressin release to minimise further water loss. However, over-activation of renal $V_{2}$ receptors contributes to cyst expansion. Although suppressing vasopressin release with high water intake has been shown to impair disease progression in rodent models, whether this approach is efficacious in patients remains un-
\end{abstract}

\begin{tabular}{ll}
\hline KARGER & $\begin{array}{l}\text { (c) } 2018 \text { The Author(s) } \\
\text { Published by S. Karger AG, Basel }\end{array}$ \\
E-Mail karger@karger.com & This article is licensed under the Creative Commons Attribution- \\
www.karger.com/anm & $\begin{array}{l}\text { NonCommercial-NoDerivatives 4.0 International License (CC BY- } \\
\text { NC-ND) (http://www.karger.com/Services/OpenAccessicense). } \\
\text { Usage and distribution for commercial purposes as well as any dis- } \\
\text { tribution of modified material requires written permission. }\end{array}$
\end{tabular}

certain. The neural osmoregulatory pathway that controls vasopressin secretion also exerts a stimulatory action on vasomotor sympathetic activity and blood pressure during dehydration. Recurrent dehydration leads to a worsening of hypertension in rodents and cross-sectional data suggests that reduced urinary concentrating ability may contribute to hypertension development in the clinical PKD population. Experimental studies are required to evaluate this hypothesis and to determine the underlying mechanism.

\footnotetext{
(c) 2018 The Author(s)

Published by S. Karger AG, Basel
}

\section{Introduction}

Polycystic kidney disease (PKD) describes a group of monogenetic disorders characterised by the acquisition of multiple renal cysts and extrarenal abnormalities including hypertension [1]. Impaired urinary concentrating ability is an early clinical presentation of PKD [2-5]. As discussed by van Gastel and Torres [6] in the proceed-

Dr. Cara M. Hildreth

Department of Biomedical Sciences

Faculty of Medicine and Health Sciences, 75 Talavera Road

Macquarie University, Sydney, NSW 2109 (Australia)

E-Mail cara.hildreth@mq.edu.au 
ings of the Hydration For Health 8th Annual Scientific Conference, physiological evidence indicates that in $\mathrm{PKD}$, this urinary concentrating defect presents a hyperosmotic stimulus to central osmoreceptors that control arginine-vasopressin (AVP) secretion. Acting at renal $V_{2}$ receptors, AVP has been shown to accelerate cystogenesis through a cyclic adenosine monophosphate-dependent mechanism [6], and interrupting this pathway (i.e., through pharmacological or genetic AVP blockade or by increasing fluid intake) can substantially attenuate cystic pathology in rodent models of PKD [7, 8]. We propose that reduced urinary concentrating capacity also facilitates the development of hypertension in PKD via osmotic stimulation of neuronal circuits in the brain that regulate blood pressure.

\section{PKD and Defective Urine Concentrating Capacity}

Two distinct forms of PKD exist that differ in their mode of inheritance and clinical presentation. Autosomal dominant $\mathrm{PKD}$ (ADPKD) is more prevalent, affecting approximately 1:1,000 individuals, and a leading cause of renal failure [1]. Though cysts are observed in children with ADPKD [1], they generally do not impair glomerular filtration until the 5th decade of life [9] (Fig. 1). Autosomal recessive PKD (ARPKD) is approximately 20 times less common, yet far more aggressive in its clinical presentation, with the majority of affected individuals showing renal insufficiency in the neonatal period [1].

The ability of the kidneys to produce concentrated urine is necessary to conserve water and therefore maintain body fluid homeostasis. Maximal urine concentrating ability is reduced in both ADPKD and ARPKD patients [2-4]. In ADPKD, this urinary concentrating impairment occurs early in the disease before renal function declines $[2,4]$ (Fig. 1), likely due to the cystic distortion of renal architecture and a consequent disruption of osmotic gradients in the renal medulla [6]. Few studies have examined the renal concentrating ability in ARPKD patients and it is unclear whether the pathophysiology is similar. Nevertheless, evidence exists that urinary concentrating ability is reduced in ARPKD patients irrespective of renal function status [4].

Cross-sectional data demonstrates that ADPKD patients with a more severe urinary concentrating defect tend to have a higher plasma osmolality, a hallmark of hypohydration [10]. Thus, a reduced ability to concentrate urine likely predisposes ADPKD patients to recurrent periods of hypohydration, a viewpoint reinforced

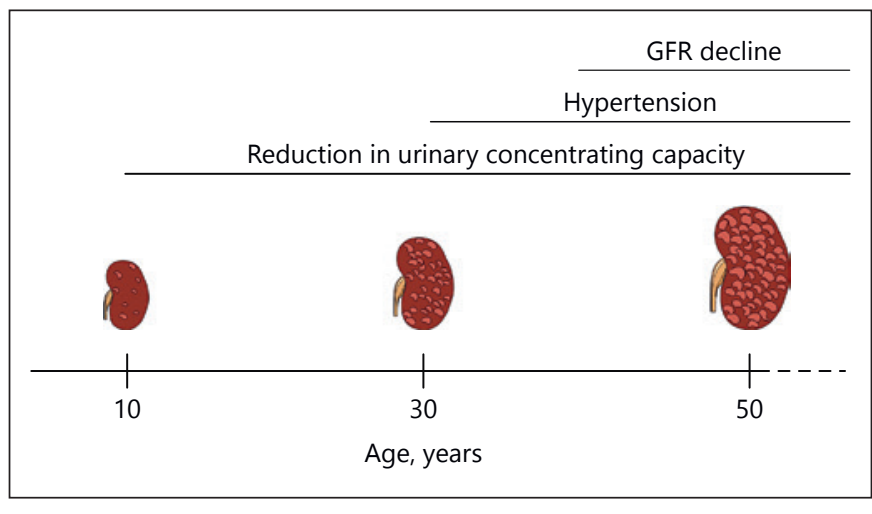

Fig. 1. Timeline illustrating the presentation of key clinical features of ADPKD in relation to age. Note that the reduction in urinary concentrating capacity precedes the development of hypertension and the decline in glomerular filtration rate (GFR). Data were acquired from $[2,8]$. Images sourced from Laboratoires Servier (www.servier.com).

by experimental evidence that consistently demonstrates that overnight water restriction produces an exaggerated increase in plasma osmolality in ADPKD patients compared to healthy individuals $[2,3]$. Whether this increased osmotic stimulus generates an increase in AVP secretion, however, has not been consistently observed. Observations by Ho et al. [2] found that overnight water restriction and a concordant increase in plasma osmolality failed to increase plasma AVP in an ADPKD clinical cohort, thus suggesting that osmoregulation of AVP secretion is impaired in ADPKD. However, this conclusion is not supported by the work of others. In ADPKD patients, Graffe et al. [11] demonstrated that intravenous hypertonic saline infusion effectively increased plasma AVP levels. Moreover, Zittema et al. [3] found that overnight water restriction in humans with ADPKD was associated with an elevation of plasma AVP and copeptin (a stable and reliable surrogate measure of AVP [5]), reaching values that were more than two-fold higher than healthy controls. It is unclear what specifically underlies these variable observations; however, it is possible that Ho et al. [2] failed to detect a significant effect because the analysis was restricted to AVP and the surrogate measure copeptin was not utilised, which is supported by evidence showing that plasma osmolality correlates with copeptin but not AVP in ADPKD patients [12]. Taken together, these studies indicate that a reduced ability to conserve body water in ADPKD most likely drives a greater activation of osmosensitive neuronal circuits in the brain that regulate AVP secretion (Fig. 2).
Underwood/Phillips/Hildreth 


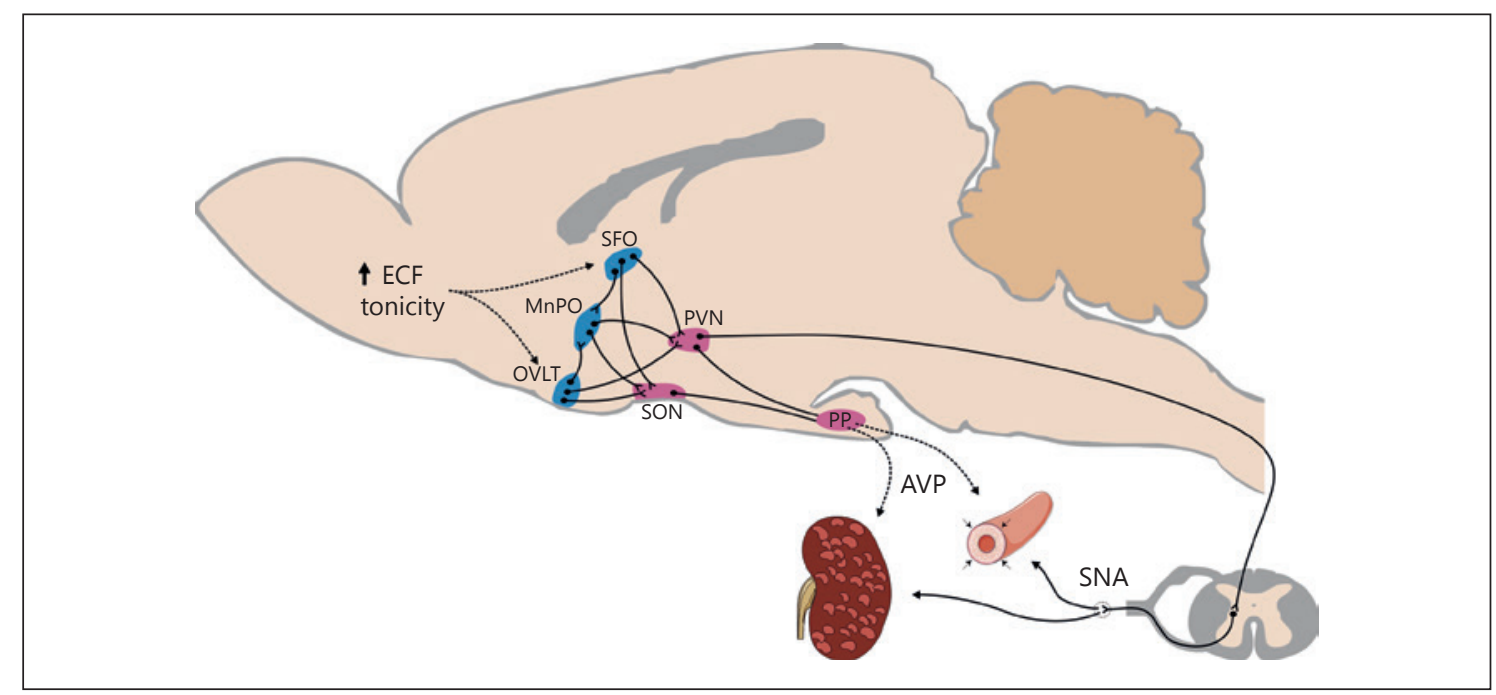

Fig. 2. Proposed neural pathway that could be recruited to accelerate renal cystogenesis and facilitate hypertension development in PKD. An early urinary concentrating impairment promotes periodic elevations in extracellular fluid (ECF) tonicity when fluid loss is not compensated by fluid intake. This is detected by osmoreceptor neurons located in 2 circumventricular organs of the lamina terminalis: the organum vasculosum of the lamina terminalis (OVLT) and the subfornical organ (SFO). The OVLT and $\mathrm{SFO}$ send direct and indirect (via the median preoptic area; $\mathrm{MnPO}$ ) projections to the paraventricular (PVN) and supraoptic (SON)

\section{Possible Role for Increased Water Intake in the Treatment of PKD}

Circulating AVP acts on renal $\mathrm{V}_{2}$ receptors to accelerate cystogenesis in PKD (for a comprehensive review see: van Gastel and Torres [6]). This was clearly demonstrated by the observation that AVP-deficient PKD rats exhibited a four-fold reduction in cyst volume that was restored upon the administration of a $\mathrm{V}_{2}$ agonist [8]. In the presence of a urinary concentrating impairment, higher plasma AVP would therefore worsen disease progression. Accordingly, clinical trials have investigated the efficacy of a $\mathrm{V}_{2}$ receptor antagonist, Tolvaptan, in ADPKD. Though clinical trials largely recapitulated the positive results shown in animal models, Tolvaptan is not without its side-effects, with safety issues noted relating to increased aquaresis and reduced liver function $[6,13]$. Alternative approaches that target the secretion of AVP rather than the $\mathrm{V}_{2}$ receptor are therefore warranted.

High water intake has been proposed as an alternative intervention to suppress AVP levels and reduce renal $V_{2}$ receptor activation in $\mathrm{PKD}$. Increasing fluid intake was found to effectively impair renal cystogenesis in rodents hypothalamic nuclei that control the peripheral release of vasopressin (AVP) from the posterior pituitary (PP) and vasomotor and renal sympathetic nerve activity (SNA). AVP accelerates renal cystogenesis via renal $\mathrm{V}_{2}$ activation and can increase blood pressure via vascular $\mathrm{V}_{1 \mathrm{a}}$ receptors. We hypothesise that recurrent exposure to ECF hypertonicity facilitates the development of hypertension by promoting a greater activity of hypothalamic neurons that control AVP secretion and SNA. Adapted from [21] with images sourced from Laboratoires Servier (www.servier.com).

[7] and has been shown to effectively decrease plasma copeptin levels in patients [14]. Though a single clinical trial did not find a beneficial effect of increasing water intake in a small cohort of ADPKD patients [15], other clinical trials with a larger number of participants and a longer treatment duration are currently underway (e.g., PREVENT-PKD trial; ACTRN12614001216606). The results of these clinical trials will be critical to evaluate whether increased water intake can suppress cystic disease progression in $\mathrm{PKD}$.

\section{Hypertension in PKD}

Hypertension is a salient feature of both ADPKD and ARPKD [1,9]. Studies in humans with ADPKD indicate that hypertension develops before renal filtration function declines in the majority of patients, suggesting that renal insufficiency is not a primary driver $[9,16]$. In these patients, hypertension contributes to cardiac left ventricular hypertrophy and renal damage [17]. A mechanistic understanding of the pathogenesis of hypertension in $\mathrm{PKD}$ remains unclear. 
One critical node underlying the hypertension observed in PKD is the renin-angiotensin system (RAS). The strongest evidence for this is that pharmacological interventions that block angiotensin II, the main effector hormone of the RAS, are effective in reducing blood pressure in patients [17]. Less clear is what tissue(s) angiotensin II acts on to produce increases in blood pressure in $\mathrm{PKD}$. The RAS is traditionally described as a circulating hormonal cascade that begins with the secretion of renin from the renal juxtaglomerular apparatus and culminates with the production of the vasopressor and natriuretic peptide angiotensin II [18]. More recent work has demonstrated that angiotensin II can be synthesised by, and act on, various tissues independent of the circulating RAS [18]. In ADPKD patients and animal models, the circulating RAS is not consistently elevated, as assessed through measurements of plasma renin concentration or enzymatic activity $[5,19]$. In contrast, urinary angiotensinogen and renin, thought to reflect the activity of the intrarenal RAS, are elevated in these patients [19]. Upregulation of the intra-renal RAS could contribute to the development of hypertension in $\mathrm{PKD}$, though causation has not been demonstrated. The brain also has a functionally-independent RAS, which serves as a critical regulator of vasomotor sympathetic nerve activity (SNA) and blood pressure [18], in part by exerting a modulatory action on the brain osmoregulatory system (see below). In ADPKD patients, the firing of sympathetic nerves supplying muscle vascular beds is elevated [20] but is reduced with angiotensin II blockers [21], supporting the hypothesis that the brain RAS is upregulated in this disease.

\section{Could Urinary Concentrating Defect Facilitate the Development of Hypertension in PKD?}

\section{Central Osmoreception and Blood Pressure Control}

Terrestrial mammals have evolved extremely sensitive regulatory mechanisms to maintain the osmolality and volume of the body fluids within narrow limits. Though the mammalian osmoregulatory system has been studied primarily in experimental animals, evidence suggests that the system functions comparably in humans [22]. During dehydration, the brain coordinates a behavioural (thirst) and physiological response that serve to buffer increased extracellular fluid osmolality and decreased intravascular volume. The physiological response consists of 2 principle effectors: AVP and the sympathetic nervous system, which together enhance renal water reabsorption, in defence of extracellular fluid osmolality, and increase vascular tone, to support arterial blood pressure [23].

The forebrain lamina terminalis is responsible for detecting plasma osmolality and initiating the behavioural and physiological response [23]. Plasma tonicity (i.e., plasma osmolality without membrane permeable solutes) is transduced by specialised osmoreceptor neurons located in 2 circumventricular organs of the lamina terminals: the organum vasculosum of the lamina terminalis (OVLT) and the subfornical organ (SFO). OVLT and SFO neurons send direct or indirect projections (via the median preoptic nucleus) to 2 hypothalamic output nuclei - the paraventricular $(\mathrm{PVN})$ and the supraoptic (SON) nuclei - that are obligatory for producing the physiological response to osmotic perturbations. These neuronal pathways are robustly activated during dehydration [24]. The PVN and SON contain magnocellular neurosecretory neurons that release AVP into the peripheral circulation, while the PVN harbors an additional population of neurons that generate vasomotor and renal sympathetic outflow [23] (Fig. 2).

Angiotensin II is an important neuromodulator in the central osmoregulatory system. In experimental animals, central hypertonicity evokes an increase in AVP secretion, sympathetic activation and rise in blood pressure that involves activation of angiotensin type 1 receptors in the brain [25-27]. Therefore, reports that systemic angiotensin II blockade reduces SNA and blood pressure in ADPKD patients [21] could reflect a role of osmoregulated brain angiotensin II in maintaining sympathoexcitation observed in this disease.

Long-term exposure to plasma hyperosmolality is strongly implicated in the development of hypertension. High salt intake is a major risk factor for hypertension. As reviewed recently by Kinsman et al. [28], longterm upregulation of the central osmoregulatory pathway described above contributes to salt-induced hypertension in rodents via sympathetic activation and AVP release. In addition to raising plasma osmolality with salt intake, a recent study by Hilliard et al. [29] showed that long-term recurrent dehydration exacerbates hypertension in rodents. This experimental evidence is concordant with cross-sectional data in humans showing that low-water drinkers $(<2.0 \mathrm{~L} /$ day $)$ are more likely to be hypertensive [30]. The underlying mechanism by which chronic hypohydration facilitates the development of hypertension is currently unknown, but could conceivably involve an increased activation or sensitisation of the central osmotic circuits regulating SNA and vasopressin secretion.
Underwood/Phillips/Hildreth 
A Possible Link between Urinary Concentrating Defect and Hypertension in PKD

There is some initial evidence to suggest that decreased urinary concentrating capacity is linked with the development of hypertension in PKD. Seeman et al. [31] found that the presence of hypertension was 7 times more common in ADPKD children and adolescents who had reduced urinary concentrating ability (maximal urine osmolality less than $900 \mathrm{mOsmol} / \mathrm{kg}$ ), and that there was a significant inverse relationship between urine osmolality and ambulatory blood pressure across the cohort. This relationship has also been observed in ADPKD adults. In a retrospective analysis of the TEMPO3/4 study, a large $(n=1,445)$ randomised control trial evaluating the efficacy of $\mathrm{V}_{2}$ receptor antagonism in ADPKD, lower urine osmolality at baseline predicted the presence of hypertension [13]. This relationship may partially explain why hypertension associates with indices of cyst abundance [16], the most likely determinant of the urine concentrating impairment.

\section{If the Relationship between Reduced Urinary}

Concentrating Capacity and Hypertension in ADPKD

Is Causal, What Could be the Underlying Mechanism?

When renal water loss is uncompensated by fluid intake, the ensuing plasma hyperosmolality would stimulate AVP secretion and vasomotor sympathetic outflow. Though an increase in blood pressure may follow, negative feedback mechanisms would maintain normotension. However, in the long term, it is possible that recurrent episodes of hypohydration sensitises blood pressure-regulating neuronal circuits, predisposing individuals to a greater risk of developing hypertension with the presentation of additional temporally separated insults (e.g., dietary factors or mental stress) later in life [18]. Conceivably, such an effect could be exacerbated by high dietary sodium. Though this hypothetical mechanism remains to be tested, work done in the Lewis PKD rat has indeed shown a marked chronic upregulation of brain regions that detect (i.e., OVLT/SFO) and respond (i.e., $\mathrm{PVN} / \mathrm{SON}$ ) to plasma hyperosmolality [32].

One obvious approach to test whether chronic hypohydration produced by the urinary concentrating defect facilitates the development of hypertension in PKD would be to determine whether increased water intake abrogates the onset or severity of hypertension in patients or animal models. Though the effects of increased water intake on the progression of PKD have been ex- amined, unfortunately hypertension was not included as an outcome measurement for the only clinical trial [15]; and the single animal study to measure blood pressure was performed on a normotensive rat strain [7]. Hence, these studies do not offer an evaluation of this hypothesis.

\section{Conclusions}

A reduced capacity to concentrate urine and consequently conserve body water presents an additional hyperosmotic stimulus for AVP release in PKD. The deleterious role of AVP in renal cystogenesis is well documented, yet whether suppression of AVP by means of increasing water intake is an effective means of halting disease progression awaits clarification. To aid in evaluating the hypothesis that hypohydration facilitates the development of hypertension in PKD, we recommend that blood pressure be reported as an outcome measure in future water intake studies.

\section{Acknowledgements}

Work in the laboratory was supported by The Hillcrest Foundation.

\section{Disclosure Statement}

C.F.U. received travel expenses and registration fee from Danone Nutricia Research to attend the 2017 Hydration for Health Scientific Conference.

\section{References}

1 Sweeney WE, Gunay-Aygun M, Patil A, Avner ED: Childhood Polycystic Kidney Disease; in Avner ED, Harmon WE, Niaudet P, Yoshikawa N, Emma F, Goldstein S (eds): Pediatr Nephrol. Berlin, Heidelberg, Springer, 2014, pp 1-58.

2 Ho TA, Godefroid N, Gruzon D, Haymann JP, Marechal C, Wang X, Serra A, Pirson Y, Devuyst O: Autosomal dominant polycystic kidney disease is associated with central and nephrogenic defects in osmoregulation. Kidney Int 2012;82:1121-1129.

3 Zittema D, Boertien WE, van Beek AP, Dullaart RP, Franssen CF, de Jong PE, Meijer E, Gansevoort RT: Vasopressin, copeptin, and renal concentrating capacity in patients with autosomal dominant polycystic kidney disease without renal impairment. Clin J Am Soc Nephrol 2012;7:906-913.
Osmoregulation in PKD: Relationship with Cystogenesis and Hypertension 
4 Kaariainen H, Koskimies O, Norio R: Dominant and recessive polycystic kidney disease in children: evaluation of clinical features and laboratory data. Pediatr Nephrol 1988;2:296302.

5 Phillips JK, Hopwood D, Loxley RA, Ghatora K, Coombes JD, Tan YS, Harrison JL, McKitrick DJ, Holobotvskyy V, Arnolda LF, Rangan GK: Temporal relationship between renal cyst development, hypertension and cardiac hypertrophy in a new rat model of autosomal recessive polycystic kidney disease. Kidney Blood Press Res 2007;30:129-144.

6 van Gastel MDA, Torres VE: Polycystic kidney disease and the vasopressin pathway. Ann Nutr Metab 2017;70(suppl 1):43-50.

7 Hopp K, Wang X, Ye H, Irazabal MV, Harris PC, Torres VE: Effects of hydration in rats and mice with polycystic kidney disease. Am J Physiol Renal Physiol 2015;308:F261-F266.

8 Wang X, Wu Y, Ward CJ, Harris PC, Torres VE: Vasopressin directly regulates cyst growth in polycystic kidney disease. J Am Soc Nephrol 2008;19:102-108.

9 Chapman AB: Approaches to testing new treatments in autosomal dominant polycystic kidney disease: insights from the CRISP and HALT-PKD studies. Clin J Am Soc Nephrol 2008;3:1197-1204.

10 Casteleijn NF, Zittema D, Bakker SJ, Boertien WE, Gaillard CA, Meijer E, Spithoven EM, Struck J, Gansevoort RT: Urine and plasma osmolality in patients with autosomal dominant polycystic kidney disease: reliable indicators of vasopressin activity and disease prognosis? Am J Nephrol 2015;41:248-256.

11 Graffe CC, Bech JN, Lauridsen TG, Pedersen EB: Urinary excretion of AQP2 and $\mathrm{ENaC}$ in autosomal dominant polycystic kidney disease during basal conditions and after a hypertonic saline infusion. Am J Physiol Renal Physiol 2012;302:F917-F927.

12 Zittema D, Casteleijn NF, Bakker SJ, Boesten LS, Duit AA, Franssen CF, Gaillard CA, Gansevoort RT: Urine concentrating capacity, vasopressin and copeptin in ADPKD and IgA nephropathy patients with renal impairment. PLoS One 2017;12:e169263.

13 Devuyst O, Chapman AB, Gansevoort RT, Higashihara E, Perrone RD, Torres VE, Blais JD, Zhou W, Ouyang J, Czerwiec FS: Urine osmolality, response to tolvaptan, and outcome in autosomal dominant poly- cystic kidney disease: results from the TEMPO 3:4 trial. J Am Soc Nephrol 2017;28: 1592-1602.

14 Amro OW, Paulus JK, Noubary F, Perrone $\mathrm{RD}$ : Low-osmolar diet and adjusted water intake for vasopressin reduction in autosomal dominant polycystic kidney disease: a pilot randomized controlled trial. Am J Kidney Dis 2016;68:882-891.

15 Higashihara E, Nutahara K, Tanbo M, Hara H, Miyazaki I, Kobayashi K, Nitatori T: Does increased water intake prevent disease progression in autosomal dominant polycystic kidney disease? Nephrol Dial Transplant 2014;29:1710-1719.

16 Gabow PA, Chapman AB, Johnson AM, Tangel DJ, Duley IT, Kaehny WD, Manco-Johnson M, Schrier RW: Renal structure and hypertension in autosomal dominant polycystic kidney disease. Kidney Int 1990;38:11771180.

17 Schrier RW, Abebe KZ, Perrone RD, Torres VE, Braun WE, Steinman TI, Winklhofer FT, Brosnahan G, Czarnecki PG, Hogan MC, Miskulin DC, Rahbari-Oskoui FF, Grantham JJ, Harris PC, Flessner MF, Bae KT, Moore $\mathrm{CG}$, Chapman AB: Blood pressure in early autosomal dominant polycystic kidney disease. N Engl J Med 2014;371:2255-2266.

18 Johnson AK, Zhang Z, Clayton SC, Beltz TG, Hurley SW, Thunhorst RL, Xue B: The roles of sensitization and neuroplasticity in the long-term regulation of blood pressure and hypertension. Am J Physiol Regul Integr Comp Physiol 2015;309:R1309-R1325.

19 Salih M, Bovee DM, Roksnoer LCW, Casteleijn NF, Bakker SJL, Gansevoort RT, Zietse R, Danser AHJ, Hoorn EJ: Urinary renin-angiotensin markers in polycystic kidney disease. Am J Physiol Renal Physiol 2017;313:F874-F881.

20 Klein IH, Ligtenberg G, Oey PL, Koomans HA, Blankestijn PJ: Sympathetic activity is increased in polycystic kidney disease and is associated with hypertension. J Am Soc Nephrol 2001;12:2427-2433.

21 Klein IH, Ligtenberg G, Oey PL, Koomans HA, Blankestijn PJ: Enalapril and losartan reduce sympathetic hyperactivity in patients with chronic renal failure. J Am Soc Nephrol 2003; 14:425-430.

22 Bourque CW: Central mechanisms of osmosensation and systemic osmoregulation. Nat Rev Neurosci 2008;9:519-531.
23 Bichet DG: Vasopressin at central levels and consequences of dehydration. Ann Nutr Metab 2016;68(suppl 2):19-23.

24 Wilson Y, Nag N, Davern P, Oldfield BJ, McKinley MJ, Greferath U, Murphy M: Visualization of functionally activated circuitry in the brain. Proc Natl Acad Sci U S A 2002;99: 3252-3257.

25 Mathai ML, Evered MD, McKinley MJ: Central losartan blocks natriuretic, vasopressin, and pressor responses to central hypertonic $\mathrm{NaCl}$ in sheep. Am J Physiol 1998;275:R548R554.

26 Watson AM, Mogulkoc R, McAllen RM, May $\mathrm{CN}$ : Stimulation of cardiac sympathetic nerve activity by central angiotensinergic mechanisms in conscious sheep. Am J Physiol Regul Integr Comp Physiol 2004;286: R1051-R1056.

27 Chen QH, Toney GM: AT(1)-receptor blockade in the hypothalamic PVN reduces central hyperosmolality-induced renal sympathoexcitation. Am J Physiol Regul Integr Comp Physiol 2001;281:R1844-R1853.

28 Kinsman BJ, Nation HN, Stocker SD: Hypothalamic signaling in body fluid homeostasis and hypertension. Curr Hypertens Rep 2017; 19:50

29 Hilliard LM, Colafella KM, Bulmer LL, Puelles VG, Singh RR, Ow CP, Gaspari T, Drummond GR, Evans RG, Vinh A, Denton KM: Chronic recurrent dehydration associated with periodic water intake exacerbates hypertension and promotes renal damage in male spontaneously hypertensive rats. Sci Rep 2016;6:33855.

30 Sontrop JM, Dixon SN, Garg AX, BuendiaJimenez I, Dohein O, Huang SH, Clark WF: Association between water intake, chronic kidney disease, and cardiovascular disease: a cross-sectional analysis of NHANES data. Am J Nephrol 2013;37:434-442.

31 Seeman T, Dusek J, Vondrak K, Blahova K, Simkova E, Kreisinger J, Dvorak P, Kyncl M, Hribal Z, Janda J: Renal concentrating capacity is linked to blood pressure in children with autosomal dominant polycystic kidney disease. Physiol Res 2004;53:629-634.

32 Ang SA, Harrison JL, Powers-Martin K, Reddrop C, McKitrick DJ, Holobotovskyy W, Arnolda LF, Phillips JK: c-Fos activation in renal hypertension. Hypertension 2007;49:14621481. 